Published in final edited form as:

Lancet Neurol. 2013 February ; 12(2): 139-148. doi:10.1016/S1474-4422(12)70311-3.

\title{
A multilevel intervention to increase community hospital use of alteplase for acute stroke (INSTINCT): a cluster-randomised controlled trial
}

\author{
Phillip A Scott, \\ Department of Emergency Medicine, University of Michigan, Ann Arbor, MI, USA \\ Stroke Program, University of Michigan, Ann Arbor, MI, USA \\ William J Meurer, \\ Department of Emergency Medicine, University of Michigan, Ann Arbor, MI, USA \\ Stroke Program, University of Michigan, Ann Arbor, MI, USA
}

\begin{abstract}
Correspondence to: Dr Phillip A Scott, Department of Emergency Medicine, University of Michigan, 24 Frank Lloyd Wright Drive, PO Box 381, Ann Arbor, MI 48109, USA phlsctt@med.umich.edu.

Contributors

PAS, SMF, JDK, MNH, RS, and LBM developed the study concept, design, and protocol. PAS obtained funding. PAS, WJM, SMF, and LBM implemented the intervention. SMF, JDK, ZX, MNH, and ZX oversaw data collection, data cleaning, merging of datasets, and preparations of extracts for analysis. JDK led the analysis with assistance from WJM and ZX. All authors contributed to interpretation of findings and preparation of the report, and approved the final version.

INSTINCT Investigators

Coordinating Center, University of Michigan: Phillip A Scott (principal investigator); Shirley M Frederiksen (project manager); William J Meurer; Allison Kade; Annette Sandretto; Lewis B Morgenstern; Cemal Sozener; Jennifer J Majersik. Biostatistics and Data Management Center, University of Michigan: John D Kalbfleisch; Mary N Haan; Zhenzhen Xu; Lana Tyrrell (project manager); Lynn Blythe (project manager); Kari Moore (data manager). Medical reviewers: Peter Panagos (Washington University, USA); Roger Humphries (University of Kentucky, USA); L Creed Pettigrew (University of Kentucky, USA). Independent Medical Monitor: Steven R Levine (State University of New York Downstate Medical Center), USA, and Kings County Hospital Center, USA).

Principal investigators, co-investigators, and coordinators at INSTINCT sites Alpena Regional Medical Center: Walter Jacquemin, Mary Jo Skiba. Battle Creek Health System: Ryan Yoder, Frank Vivio. Borgess Medical Center: Rashmi Kothari, Christine McNett. Botsford Hospital: Sanford J Vieder. Community Health Center of Branch County: Marion Labadie, Ted Gould, David Fuchs. Covenant Healthcare: Brian Schroeder, Joseph Spadafore. Holland Hospital: Sandip Kothari, Jan Culina-Troeger, Joseph Bonello. Lakeland Community Hospital: Michael Westfall. Lakeland Regional Medical Center: Robert Ward 3rd. Lapeer Regional Medical Center: Bradley Blaker, Ralph Scolari, David Kraus. Memorial Healthcare: Ronald Bishop. Mercy Health Partners, Hackley Campus: Kevin Kellogg, Tod Wyn, Angela Gilmore. Mercy Hospital Cadillac: Sally A Ancel, Alan Kennedy, Joanna Warner. Mercy Memorial Hospital: Donald Brock, Stephen Grider, Tina Melonakos. Munson Medical Center: Kenneth Friar, William Chung, Kathleen Glaza. McLaren Northern Michigan: Matthew Bowes, Elaine Siwiec. Oakwood Southshore Medical Center: Arash Armin, David R Weaver. Port Huron Hospital: Michael K Paul, Daniel Angeli. Saint Mary's Health Care: Michael Olgren, Darryl Varda, Tami Nysse, Julie Levandoski, Karen Burritt. Sparrow Hospital and Health System: Arshad Majid, Mary Lou Mitchell, Susan Wehner. St John Hospital and Medical Center: Patricia P Nouhan. St John River District Hospital: Ronald Thies, Lanying Brown. West Shore Medical Center: Richard Scharp. William Beaumont Hospital-Troy: Aveh Bastani, Sydney Leach, Rosemarie HuntWalch, Cindy Huckabone, Blerina Shaqiri.

Conflicts of interest

PAS receives grant funding from the National Institutes of Health (NIH) and the Michigan Department of Community Health. PAS also provides paid expert medical-legal review for Beck and Amsden PLLC; Robbins, Kaplan, Miller and Ciresi LLP; Williams Venker and Sanders LLC Attorneys at Law; and Hinshaw and Culbertson LLP. WJM receives grant funding from the NIH and Agency for Healthcare Research and Quality, and receives payment for statistics review by Academic Emergency Medicine. WJM also provides paid expert medical-legal review for Smith, Haughey, Rice and Roegge Attorneys at Law, and Reminger Attorneys at Law. SMF, JDK, ZX, MNH, and RS receive grant funding from the NIH. LBM receives grant funding from the NIH and was a paid consultant for Genentech on one occasion during the study.

See Online for appendix

For the trial protocol see http://medicine.umich.edu/sites/default/files/content/downloads/INSTINCT1\%20Clinical\%20Protocol \%20Amend2_03_10_08.pdf

For specific lectures see http://www.med.umich.edu/em/research/instinct/index.htm
\end{abstract}


Department of Neurology, University of Michigan, Ann Arbor, MI, USA

Shirley M Frederiksen,

Department of Emergency Medicine, University of Michigan, Ann Arbor, MI, USA

Stroke Program, University of Michigan, Ann Arbor, MI, USA

John D Kalbfleisch, Department of Biostatistics, University of Michigan, Ann Arbor, MI, USA

Zhenzhen Xu,

Department of Biostatistics, University of Michigan, Ann Arbor, MI, USA

Mary N Haan,

Department of Epidemiology, University of Michigan, Ann Arbor, MI, USA

Department of Epidemiology and Biostatistics, University of California, San Francisco, San Francisco, CA, USA

Robert Silbergleit, and

Department of Emergency Medicine, University of Michigan, Ann Arbor, MI, USA

Stroke Program, University of Michigan, Ann Arbor, MI, USA

Lewis B Morgenstern

Department of Emergency Medicine, University of Michigan, Ann Arbor, MI, USA

Stroke Program, University of Michigan, Ann Arbor, MI, USA

Department of Neurology, University of Michigan, Ann Arbor, MI, USA

Department of Epidemiology, University of Michigan, Ann Arbor, MI, USA

\section{the INSTINCT Investigators}

\section{Summary}

Background-Use of alteplase improves outcome in some patients with stroke. Several types of barrier frequently prevent its use. We assessed whether a standardised, barrier-assessment, multicomponent intervention could increase alteplase use in community hospitals in Michigan, USA.

Methods-In a cluster-randomised controlled trial, we selected adult, non-specialty, acute-care community hospitals in the Lower Peninsula of Michigan, USA. Eligible hospitals discharged at least 100 patients who had had a stroke per year, had less than 100000 visits to the emergency department per year, and were not academic comprehensive stroke centres. Using a computergenerated randomisation sequence, we selected 12 matched pairs of eligible hospitals. Within pairs, the hospitals were allocated to intervention or control groups with restricted randomisation in January, 2007. Between January, 2007, and December, 2007, intervention hospitals implemented a multicomponent intervention that included qualitative and quantitative assessment of barriers to alteplase use and ways to address the findings, and provided additional support. The primary outcome was change in alteplase use in patients with stroke in emergency departments between the pre-intervention period (January, 2005, to December, 2006) and the post-intervention period (January, 2008, to January, 2010). Physicians in participating hospitals and the coordinating centre could not be masked to group assignment, but were masked to progress made in paired control hospitals. External medical reviewers who were masked to group assignment assessed outcomes. We did intention-to-treat (ITT) and target-population (without one pair that was excluded after randomisation) analyses. This trial is registered at ClinicalTrials.gov, number NCT00349479. 
Findings-All 24 hospitals completed the study. Overall, 745 of 40823 patients with stroke received intravenous alteplase treatment. In the ITT analysis, the proportion of patients with stroke who were admitted and treated with alteplase increased between the pre-intervention and postintervention periods in intervention hospitals ( 89 [1.25\%] of 7119 patients to 235 [2.79\%] of 8419) to a greater extent than in control hospitals (99 [1.25\%] of 7946 to 194 [2.10\%] of 9222), but the difference between groups was not significant (relative risk [RR] 1.37, 95\% CI 0.96-1.93; $\mathrm{p}=0.08)$. In the target-population analysis, the increase in alteplase use in intervention hospitals (59 [1.00\%] of 5882 to 191 [2.62\%] of 7288) was significantly greater than in control hospitals (65 [1.09\%] of 5957 to 120 [1.72\%] of 6989 ; RR $1.68,95 \%$ CI 1.09-2.57; p=0.02), but was still clinically modest.

Interpretation-The intervention did not significantly increase alteplase use in patients with ischaemic stroke. The increase in use of alteplase in the target population was significant, but smaller than the effect to which the study was powered. Additional strategies to increase acute stroke treatment are needed.

\section{Introduction}

Worldwide, stroke is the second leading cause of preventable death and the fourth leading cause of adult disability. ${ }^{1}$ Although intravenous tissue plasminogen activator (alteplase) increases the chance of a good outcome in some patients,, 3 this treatment is underused. Estimates suggest that, although up to $11.5 \%$ of patients with stroke are eligible, $2 \%$ or less receive thrombolytics. ${ }^{4,5}$ If delays in patient and physician recognition and response to the signs and symptoms of acute stroke could be eliminated, the proportion of patients with stroke who are eligible for alteplase could be as high as $24 \%{ }^{6}$

Designated stroke centres (ie, hospitals with specific accreditation to deliver stroke treatment) have improved alteplase delivery. However, less than one in four patients in the USA live where they could travel to one of these centres by land within $30 \mathrm{~min} .{ }^{7}$ In view of the short treatment window in which alteplase can be given to individuals with stroke, many patients present to local community hospitals. Opportunities for effective treatment in these settings are poor. A national review showed that $64 \%$ of community hospitals in the USA reported no alteplase treatments of acute stroke between 2005 and 2007. ${ }^{8}$ Data suggest that neurologists are infrequently involved with acute stroke care in community emergency departments, and emergency physicians have expressed concerns about barriers to thrombolytic treatment (time, resources, organisation, and personal experience) and risk of haemorrhagic complications. ${ }^{9-12}$ Similar difficulties in geographical and specialist access have been reported in other countries. ${ }^{13,14}$

A previous study ${ }^{15,16}$ showed that sustained increases in community alteplase delivery were possible with a rational behavioural intervention targeting both patients and providers. However, no randomised controlled trial has tested a practical intervention to increase stroke thrombolytic delivery in a representative sample of community hospitals as far as we are aware. Identification of a successful strategy could improve stroke care and serve as a model to enhance adoption of other high-risk treatments.

The aim of the INcreasing Stroke Treatment through INterventional Change Tactics (INSTINCT) trial was to assess the ability of a multilevel, barrier assessment-interactive educational intervention (BA-IEI) to increase alteplase use in community hospitals in Michigan, USA. We postulated that identification and addressing of local barriers to alteplase use in patients with stroke would increase thrombolytic use at intervention hospitals compared with matched control hospitals. 


\section{Methods}

\section{Study design}

The INSTINCT trial was a cluster-randomised controlled trial. Because hospital personnel and systems were the target of the intervention, hospitals were used as the unit of randomisation to minimise contamination. The Biostatistics and Data Management Center (BDMC) at the University of Michigan, which is independent of the INSTINCT Clinical Coordinating Center, selected community hospitals from the list of 104 adult, non-specialty, acute-care hospitals in the Lower Peninsula of Michigan. We excluded hospitals that discharged fewer than 100 patients with a diagnosis of stroke per year, had more than 100 000 visits to the emergency department per year, were an academic comprehensive stroke centre, ${ }^{17}$ or were affiliated with the University of Michigan coordinating centre.

From the pool of eligible hospitals, the BDMC selected an index hospital using a computergenerated randomisation sequence (SAS version 9.1). A matching hospital, in which the number of patients discharged after stroke per year was within $20 \%$ of that of the index hospital, was then randomly selected using a computer generated randomisation sequence. Once a hospital was selected (either as index or the match) all hospitals within 15 miles were excluded from the sampling pool. This process was repeated until 12 hospital pairs (24 hospitals) were obtained. Hospital selection was completed in 2004.

We obtained institutional review board approval at all sites. Written informed consent or waiver of documentation of consent was obtained for participating physicians and hospital staff. Review of patient records was completed under an approved Waiver or Alteration of Consent. Consent for treatment with alteplase was obtained from patients in accordance with local hospital standards.

\section{Randomisation and masking}

Randomisation was completed by the BDMC in January, 2007. Assignment to intervention or control group was determined with a computer-generated randomisation sequence (SAS version 9.1) with a 50:50 chance. The assignment status within three pairs was reversed by the BDMC to reduce confounding by achieving greater balance between the number of hospitals in northern and southern Michigan (reflecting urban and rural areas, appendix). This process also balanced the groups for the percentage of black people and the percentage who were younger than 65 years in the surrounding population. US Census Bureau data (2000) were used to obtain relevant demographic information. All hospitals and the coordinating centre were notified of group assignments simultaneously. Masking of study and hospital personnel to site assignment was not possible because of the nature of the intervention. Participating hospital staff and clinical investigators implementing inter ventions were masked to progress in the paired control site and to all interim analyses until study completion. External medical reviewers who were masked to group assignment assessed outcomes and appropriateness of alteplase use.

\section{Procedures}

Hospitals assigned to the intervention group implemented the BA-IEI from January to December 2007, with some scheduled recurrent activities continuing until trial completion. The intervention was designed to alter systems and behaviour at an institutional level and individual stafflevel, focusing on change in the emergency department setting. It was based on behaviour change theory and adapted from previous experience in the development of alteplase delivery systems. ${ }^{15,18}$ The BA-IEI included qualitative and quantitative assessment of barriers to alteplase use combined with several elements to address the findings: clinical practice guideline promotion, development of local stroke champions (ie, an opinion leader 
within a community who was deemed likely to influence the acceptance of an innovation or practice by that community), continuing education, telephone support for treatment decisions, academic detailing (ie, non-commercial education of health-care professionals about the evidence-based efficacy, safety, and cost of therapies in a non-biased way, with discussion of the entire range of treatment options), and audit and feedback mechanisms (table 1). ${ }^{19}$ Additional stroke treatment support was also provided as part of the BA-IEI. Control hospitals received no intervention and were to continue as normal.

The primary outcome was the difference in alteplaseuse in patients with stroke in emergency departments between the pre-intervention period (January, 2005, to December, 2006) and the post-intervention period (January, 2008, to January, 2010). We measured change in alteplase use as the change in absolute counts of patients treated with alteplase; this difference was analysed with adjustment for the total number of patients admitted with ischaemic stroke (irrespective of alteplase eligibility) to standardise the measure of alteplase use. Secondary outcomes were measurements of safety (any intracerebral haemorrhage within 10 days, symptomatic intracerebral haemorrhage within $36 \mathrm{~h}$, and major sys temic haemorrhage); appropriateness of alteplase use; and change in physician knowledge, beliefs, and attitudes towards alteplase use (to be reported separately).

Use of alteplase for stroke was established monthly with pharmacy data, stroke logs, and the International Classification of Diseases 9 code for infusion of a thrombolytic agent (ICD-9; 99.10). Every case was verified at the hospital site with medical records by personnel from the coordinating centre. From the start of the intervention stage, de-identified medical records were forwarded to three independent, external physician reviewers masked to hospital assignment to establish the appropriateness of alteplase use and to identify treatment complications. Appropriate use was defined a priori with the 2005 American Heart Association guidelines, ${ }^{20}$ product labels approved by the US Food and Drug Administration, and the inclusion and exclusion criteria from the National Institute of Neurological Disorders and Stroke alteplase stroke study (appendix). ${ }^{2}$ The total number of patients discharged after stroke was established four times a year with ICD-9 codes previously validated for passive surveillance of ischaemic cerebrovascular disease $(434,435,436$, 437.1, and 437.6). ${ }^{21}$

\section{Statistical analysis}

We compared intervention and control groups, taking account of correlations within hospitals and within pairs, with an otherwise unadjusted intention-to-treat (ITT) analysis as the primary analysis. In 2007, after randomisation, a control hospital became an academic comprehensive stroke centre with a neurovascular stroke fellowship (ie, a study entry exclusion criterion). Before study completion or data analysis, we decided to do a targetpopulation analysis excluding this hospital and its matched pair. This target-population analysis focused on the population of interest—community hospitals—-for which the intervention was designed and complete data were available.

The study was conservatively powered with the assumption that ten pairs of eligible hospitals would be available for analysis. Additionally, we assumed that 1 year of preintervention data and 2 years of post-intervention data would be available for comparison. Of specific interest in calculation of sample size was the difference in the percentage change in alteplase use between control and intervention sites. We calculated sample size and power and did subsequent analyses with methods appropriate for the cluster-randomised design of the trial. If mean difference between the change in alteplase use from the pre-intervention period to the post-intervention period in the intervention hospitals and that in the control hospitals was as large as $3.5 \%$, the study would have a power of about $97 \%$ at the $5 \%$ significance level with a two-tailed test. The study would have an $83 \%$ power to detect a $3 \%$ 
difference. The recruited sample size was increased to twelve hospital pairs, with 2 years of pre-intervention data, to protect against hospital-pair loss. Both these changes would increase the power of the study.

We used a mixed-effects Poisson regression model for the frequency of alteplase use, with the number of strokes as an offset, to evaluate differences between the intervention and control groups. The pre-intervention period was the reference period for both time and treatment, and the model assumed no treatment difference existed before randomisation, as preliminary investigations confirmed. This model formed the basis of our primary analysis, in which the effect of the intervention was measured by the contrast between postintervention and pre-intervention frequency of alteplase use in all patients admitted with ischaemic stroke (irrespective of eligibility for alteplase). This general model also allowed for a separate random effect of each hospital and a random effect of each matched pair to adjust for the correlations between repeated measurements of alteplase use in each hospital across time and correlations between members in each matched pair. The intraclass correlation coefficientis applicable to situations in which the response of interest within the clusters is roughly normally distributed. We did, however, assume an intrahospital correlation between the alteplase treatment rates in the pre-intervention and postintervention periods of $0 \cdot 25$. We used the Generalized Linear Mixed Model procedure in SAS (version 9.2; SAS Corporation, Cary, NC, USA).

An independent medical safety monitor assessed unblinded data after 80, 160, and 240 treatments in the intervention group to assess safety and performance.

This trial is registered at ClinicalTrials.gov, number NCT00349479.

\section{Role of the funding source}

The sponsors of the study had no role in study design, data collection, data analysis, data interpretation, or writing of the report. The corresponding author had full access to all the data in the study, and PAS and JDK had final responsibility for the decision to submit for publication.

\section{Results}

Of the initial 24 hospitals selected, five declined to participate (figure 1); we randomly selected five replacements from the list of eligible hospitals. All 24 hospitals assigned completed the study (figure 1). These hospitals represented 18\% (24 of 137) of all adult, acute-care hospitals in Michigan and 39\% (24 of 61) of all eligible hospitals. The pair of hospitals excluded after assignment in the target-population analysis were two of the largest hospitals in the sample and together accounted for 153 alteplase treatments after randomisation. Table 2 shows the characteristics of all hospitals and their emergency physician staff by group assignment and comparison study period.

Barriers to acute stroke treatment have previously been reported. ${ }^{12}$ In our study, barriers were characterised as external or internal to the treating physician. External barriersparticularly factors relating to the hospital environment and the patients-dominated in every hospital. We identified several specific issues: communication with radiology teams, poor availability of neurologists, and delay in presentation and symptom recognition. Treatment guideline familiarity and physician motivation were perceived as the primary barriers internal to the emergency department. All these barriers-with the exception of presentation delay—were addressed by one or more intervention elements. 
All intervention group hospitals participated in every element of the BA-IEI. 542 hospital staff attended sessions of continuing medical education, 233 parti cipated in the first two mock stroke codes, and a mean of 3.7 staff per intervention hospital (SD 1.9) attended the annual educational stroke champions meetings in Ann Arbor (MI, USA). Telephone support was requested for 165 patients from 12 intervention sites, resulting in a recommendation to treat or possibly treat in $102(62 \%)$ cases. 77 incidents requiring critical incident defusing sessions were identified in 74 patients from 11 intervention sites; all sessions were completed. No interventions were implemented at control sites; however, one telephone support request originated from a control hospital, probably during a routine transfer of a patient with a stroke.

In all hospital pairs and study phases, 745 (1.8\%) of 40823 patients with stroke received intravenous alteplase treatments. In the pre-intervention phase, $188(1.2 \%)$ of 15065 patients with stroke were given alteplase. We obtained detailed treatment and safety data for the patients with stroke who received alteplase (557 [2.2\%] of 25758 ) in the intervention and post-intervention phases.

In the ITT analysis, the mean number of patients treated with alteplase per month increased from 3.7 (SD 1.9) in the pre-intervention period to 9.4 (3.0) in the post-intervention period in intervention hospitals, and from $4.1(2.4)$ to $7 \cdot 8(3.5)$ in control hospitals.

The percentage of patients treated with alteplase among all those admitted with stroke increased between the pre-intervention and post-intervention phases, from 1.25\% (89 of 7119 ) to $2.79 \%$ ( 235 of 8419 ) in the intervention group and from $1.25 \%$ (99 of 7946 ) to $2.10 \%$ (194 of 9222) in the control group. The difference between the change in the intervention group $(1 \cdot 54 \%, 95 \%$ CI $1 \cdot 1-2 \cdot 0$, absolute increase; $123 \%$ relative increase) and the change in the control group $(0.85 \%, 0.47-1 \cdot 2$, absolute increase; $68 \%$ relative increase) was $0.69 \%$ ( $55 \%$ difference in relative increase).

However, the difference was not significant in the comparison based on the mixed-effects Poisson model (relative risk [RR] 1.37, 95\% CI 0.96-1.93; $\mathrm{p}=0.08$; table 3). Assessment of individual hospital data (figure 2) showed that, of the five hospitals achieving an improvement of at least 3.5\% from their baseline alteplase treatment rate (the prespecified clinically important improvement), four were from the inter vention group. Similarly, in examining maximum treat ment rates, four of five hospitals in which between 5\% and $8 \%$ of all ischaemic strokes were treated with alteplase were from the intervention group (figure 2).

In the hospitals included in the target-population analysis, 124 (1.0\%) of 11839 patients with stroke received alteplase in the pre-intervention phase. In the intervention and postintervention phases, 404 (2.0\%) of 20698 patients with stroke received alteplase. The mean number of patients treated with alteplase per month increased from 2.5 (SD 1.3) in the preintervention period to $7.6(2.5)$ in the post-intervention period in intervention hospitals, and from $2.7(1.8)$ to $4.8(2.4)$ in control hospitals.

In the target population, the percentage of patients treated with alteplase among all those admitted with stroke increased between the pre-intervention and post-intervention phases, from $1.00 \%$ (59 of 5882) to $2.62 \%$ (191 of 7288) in the intervention group, and from $1.09 \%$ (65 of 5957) to $1.72 \%$ (120 of 6989) in the control group. The difference between the change in the intervention group $(1.62 \%$ [95\% CI 1.2-2.1] absolute increase; $162 \%$ relative increase) and the change in the control group (0.63\% [0.22-1.03] absolute increase; $58 \%$ relative increase) was $0.99 \%$ (104\% difference in relative increase). This difference was significant in the com parison based on the mixed-effect Poisson model (RR 1.68, 95\% CI $1 \cdot 09-2 \cdot 57 ; \mathrm{p}=0.02$; table 3 ). 
In the ITT population, 549 (98.6\%) of the 557 patients who received alteplase during the intervention and post-intervention periods underwent post-treatment neuro-imaging. 94 $(16.9 \%)$ were identified as having evidence of post-treatment intracranial bleeding of any degree within 10 days of treatment. $33(5.9 \%)$ had symptomatic intracranial haemorrhage within $36 \mathrm{~h}$ of treatment (17 [5.6\%] of 302 in intervention hospitals; 16 [6.3\%] of 255 in control hospitals; $p=0.73)$. In the target-population analysis, 403 (99.8\%) of the 404 patients who received alteplase during the intervention and post-intervention periods underwent post-treatment neuroimaging, $24(5.9 \%)$ patients had symptomatic intracranial haemorrhage within $36 \mathrm{~h}(15$ [6.1\%] of 244 in intervention hospitals; nine [5.6\%] of 160 in control hospitals; $\mathrm{p}=0 \cdot 82$ ).

$14(2.5 \%)$ patients in the ITT population who received alteplase had a systemic haemorrhage requiring two or more units of blood products (six [2.0\%] in intervention hospitals; eight [3.1\%] in control hospitals; $\mathrm{p}=0 \cdot 40) .62(11.1 \%)$ patients died in hospital (33 of 302 [10.9\%] in intervention hospitals; 29 of 255 [11.4\%] in control hospitals; $\mathrm{p}=0.84)$.

The masked medical reviewers deemed that all 557 patients in the ITT population who received alteplase had a clinical diagnosis of stroke at the time of treatment. At discharge, ten $(1.8 \%)$ patients had an alternative documented diagnosis (three seizure, three conversion disorder, two complicated migraine, one multiple sclerosis, and one intoxication).

$152(27 \cdot 3 \%)$ patients who received alteplase had at least one identified pretreatment deviation from appropriate use guidelines: $81(26.8 \%)$ of 302 in intervention hospitals and $71(27.8 \%)$ of 255 in control hospitals. $18(3.2 \%)$ had more than one pretreatment deviation. The most common deviations were treatment after $3 \mathrm{~h}$ (43 [14.2\%] in intervention hospitals; $39[15.3 \%]$ in control hospitals) and failure to document blood pressure within the necessary range before treatment (16 [5.3\%] in intervention hospitals; 23 [9.0\%] in control hospitals). Post-treatment deviations were more frequent than were pretreatment deviations: 213 (38.2\%) patients had at least one identified post-treatment deviation (126 [41.7\%] in intervention hospitals; 87 [34.1\%] in control hospitals) and 31 (5.6\%) had more than one deviation. Common post-treatment deviations were blood pressure not maintained within specified limits (85 [28.1\%] in intervention hospitals; 60 [23.5\%] in control hospitals) and premature (within $24 \mathrm{~h}$ ) use of antiplatelet or antithrombotic agents (36 [11.9\%] in intervention hospitals; 23 [9.0\%] in control hospitals). No differences in the numbers of deviations from guidelines between intervention and control hospitals were significant in ITT or target-population analyses.

We assessed appropriate use for the decision to initiate alteplase treatment, the subsequent $24 \mathrm{~h}$ of care, and both combined. Because detailed data for appropriate use were available only after randomisation, we compared the intervention and post-intervention periods. The increase in the proportion of patients admitted for stroke and appropriately treated with alteplase between the intervention and post-intervention periods was greater in intervention hospitals than in control hospitals (absolute $0.66 \%$ vs $0.35 \%$, difference $0.31 \%$; relative $112 \%$ vs $46 \%$, difference $66 \%$ ). However, the difference was not significant in ITT or target-population analyses (appendix).

We located, de-identified, abstracted, and analysed medical records for all 557 patients treated with alteplase after randomisation according to protocol (100\% capture). Overall, 99 066 (99\%) of 100260 individual data fields sought were obtained. Inter-rater agreement for coordinator abstracted elements was 94\% (2049 of 2170) overall and 96\% (374 of 390) for a-priori identified critical elements. Inter-rater agreement between physician reviewers was 98\% (17 383 of 17824 ). 


\section{Discussion}

Despite the use of comprehensive barrier assessments and targeted multilevel interventions, our pragmatic intervention did not significantly increase alteplase use in patients with ischaemic stroke. The data suggest that the intervention had a smaller, more heterogeneous effect than was that for which the study was powered. A significant increase in the proportion of patients treated with alteplase per month was identified in the targetpopulation analysis. Possible explanations for the modest effect size are limitations of the intervention itself, local hospital events, and the effect of secular trends.

Benefits for patients are maximised when effective treatments identified by clinical research are subsequently adopted in routine clinical practice and decision making-a process known as second-stage knowledge translation. However, alteration of health-care-provider practice is hard. Our trial emphasises the challenge of creation of pragmatic interventions to improve knowledge translation to safely increase rates of alteplase use in patients with stroke.

The BA-IEI was designed for distribution by academic comprehensive stroke centres to a few regional hospitals annually as part of their educational outreach mission. ${ }^{17}$ This design enhanced generalisability but restricted the knowledge translation effort that was possible. The BA-IEI included four of the six identified methods to change physician behaviour (education, feedback, physician participation, and administrative oversight). The two methods that were not used were financial incentives and disincentives. ${ }^{22}$ The effort or elements might have been insufficient to effect the desired change. Although the intervention primarily targeted the emergency department, it included personnel and systems throughout the hospital environment. We targeted emergency departments because they are the primary location of alteplase treatment in US hospitals, and because emergency physicians and their professional organisations had limited enthusiasm for its use in stroke. ${ }^{10,11}$

Local events could also have affected the results. Imbalances in hospital expansion (a disruptive process) and loss of emergency physicians trained during the study (affecting the intervention group only) were identified and favoured the control hospitals. An imbalance in the presence of primary stroke centres also favoured the control hospitals in all study periods. The inclusion of primary stroke centres in the study cohort was intentional, because many hospitals that are designated as primary stroke centres have little experience of alteplase use, particularly immediately after certification. ${ }^{23}$ However, status as a primary stroke centre is associated with a strong institutional commitment to acute stroke care. These events and imbalances potentially increased the difficulty in identification of a difference between groups.

In the USA, a strong secular trend towards increasing alteplase use has emerged, with a doubling of treatment rates between 2005 and 2010, after a decade of little or no improvement. ${ }^{5,24}$ This unexpected trend was probably driven by several factors: the 2005 approval of increased reimbursement for thrombolytic treatment of stroke; the 2008 report of the European Cooperative Acute Stroke Study (ECASS) III trial results ${ }^{3}$ supporting the efficacy of alteplase treatment; the emergence of primary stroke centres; ${ }^{25}$ expansion of telemedicine; and efforts by the American Heart Association's Get With The Guidelines programme to improve stroke care. ${ }^{26}$

The heterogeneity of the response to the intervention suggests that local conditions might greatly affect results of knowledge-translation efforts. Findings suggesting only minimal improvement in aggregate data should be interpreted with caution. Additionally, an assessment of change in emergency physician knowledge about stroke treatment showed a 
significant improvement in understanding of alteplase eligibility and treatment delivery for the intervention hospitals compared with control hospitals (data to be reported elsewhere).

Importantly, INSTINCT provides the first assessment of safety of stroke thrombolysis in a diverse, randomly selected cohort of hospitals with sufficient treatment numbers to ensure precise safety metrics. The data confirm that the rate of intracranial haemorrhage is consistent with that in efficacy trials in which alteplase was given by personnel with stroke expertise. ${ }^{2,3,27}$ These safety outcomes were achieved despite a fairly high number of deviations from strict interpretation of reported guidelines for appropriate use. The most common pretreatment deviation identified was time to treatment ( $\leq \mathrm{h}$ ) - a measure that was confounded by the ECASS III trial report during the post-intervention phase. ${ }^{3}$ The ECASS III results showed that the effective stroke treatment window could be extended to $4.5 \mathrm{~h}$ without a meaningful increase in risk of intracranial haemorrhage; they probably affected treatment decisions within our trial. Data from the third international stroke trial (IST-3), ${ }^{28}$ published in 2012, also support the notion that time to treatment within a $4.5 \mathrm{~h}$ window has little effect on bleeding complications.

Our findings should have substantial generalisability in view of the methods used in hospital selection and the pragmatic nature of the intervention and outcome measures. Hospitals were in urban, suburban, and rural locations; were of a broad spectrum in size; and had a diverse population of patients. As such, we would expect the results to represent a broad crosssection of US hospitals and potentially other similar sized and resourced hospitals in other developed communities worldwide. We believe that our study could inform health-care systems, including those with well developed systems for stroke care, about the effort necessary for expansion of thrombolytic delivery and what degree of success is likely (panel). Finally, INSTINCT provides key insights into the effort necessary for, and limitations of, knowledge translation for high-risk interventions in the community setting.

Our trial has several limitations. First, we did not seek information about untreated patients. To obtain such data would have used resources beyond the scope of the trial and potentially duplicated other research efforts directly addressing questions about population eligibility for alteplase. ${ }^{33}$ Thus, our data do not provide information about the overall treatment opportunities available. Second, the design did not allow assessment of 90-day functional outcomes that would have allowed direct outcome comparison with efficacy trials of stroke thrombolysis. Third, our methods precluded assessment of the degree each individual BAIEI element contributed to the intervention. Fourth, baseline characteristic data of patients treated with alteplase were not gathered in the pre-intervention period, restricting comparisons. Finally, use of ICD-9 codes for surveillance of patients with stroke has known limitations in accuracy, but was resource efficient and consistent.

In summary, the INSTINCT intervention did not significantly increase alteplase use in patients with ischaemic stroke in the ITT population. Differences in treatment rates in the target population were significant, but were smaller than the effect to which the study was powered. Additional strategies to increase acute stroke treatment are needed.

\section{Supplementary Material}

Refer to Web version on PubMed Central for supplementary material.

\section{Acknowledgments}

This trial was supported by grant number R01-NS-050372 from the National Institutes of Health (NIH) National Institute of Neurological Disorders and Stroke. The content is solely the responsibility of the authors and does not necessarily represent the official views of the NIH. No other compensation was provided except that from the study 
sponsor. We gratefully acknowledge the many contributions of time and talent from the physicians and staffat the participating hospitals and coordinating centre to identify methods to improve stroke care.

Funding National Institutes of Health National Institute of Neurological Disorders and Stroke.

\section{References}

1. Lopez AD, Mathers CD, Ezzati M, Jamison DT, Murray CJ. Global and regional burden of disease and risk factors, 2001: systematic analysis of population health data. Lancet. 2006; 367:1747-57. [PubMed: 16731270]

2. The National Institute of Neurological Disorders and Stroke rt-PA Stroke Study Group.Tissue plasminogen activator for acute ischemic stroke. N Engl J Med. 1995; 333:1581-87. [PubMed: 7477192]

3. Hacke W, Kaste M, Bluhmki E, et al. Thrombolysis with alteplase 3 to 4.5 hours after acute ischemic stroke. N Engl J Med. 2008; 359:1317-29. [PubMed: 18815396]

4. Hills NK, Johnston SC. Why are eligible thrombolysis candidates left untreated? Am J Prev Med. 2006; 31(suppl 2):S210-16. [PubMed: 17178305]

5. Kleindorfer D, Lindsell CJ, Brass L, Koroshetz W, Broderick JP. National US estimates of recombinant tissue plasminogen activator use. Stroke. 2008; 39:924-28. [PubMed: 18239184]

6. Boode B, Welzen V, Franke C, van Oostenbrugge R. Estimating the number of stroke patients eligible for thrombolytic treatment if delay could be avoided. Cerebrovasc Dis. 2007; 23:294-98. [PubMed: 17199087]

7. Albright KC, Branas CC, Meyer BC, et al. ACCESS: acute cerebrovascular care in emergency stroke systems. Arch Neurol. 2010; 67:1210-18. [PubMed: 20937948]

8. Kleindorfer D, Xu Y, Moomaw CJ, Khatri P, Adeoye O, Hornung R. US geographic distribution of rt-PA utilization by hospital for acute ischemic stroke. Stroke. 2009; 40:3580-84. [PubMed: 19797701]

9. Brown DL, Lisabeth LD, Garcia NM, Smith MA, Morgenstern LB. Emergency department evaluation of ischemic stroke and TIA: the BASIC Project. Neurology. 2004; 63:2250-54. [PubMed: 15623682]

10. American College of Emergency Physicians. [Dec 9, 2012] Policy compendium 2012: Use of intravenous tPA for the management of acute stroke in the emergency department. 2002. p. 110http://www.acep.org/ Physician-Resources/Policies/2012-Policy-Compendium/

11. Brown DL, Barsan WG, Lisabeth LD, Gallery ME, Morgenstern LB. Survey of emergency physicians about recombinant tissue plasminogen activator for acute ischemic stroke. Ann Emerg Med. 2005; 46:56-60. [PubMed: 15988427]

12. Meurer WJ, Majersik JJ, Frederiksen SM, Kade AM, Sandretto AM, Scott PA. Provider perceptions of barriers to the emergency use of tPA for acute ischemic stroke: a qualitative study. BMC Emerg Med. 2011; 11:5. [PubMed: 21548943]

13. Aaron S, Alexander M, Maya T, Mathew V, Goyal M. Treatment of acute ischemic stroke: awareness among general practitioners. Neurol India. 2010; 58:441-42. [PubMed: 20644275]

14. Rudd M, Rodgers H, Curless R, et al. Remote specialist assessment for intravenous thrombolysis of acute ischaemic stroke by telephone. Emerg Med J. 2012; 29:704-08. [PubMed: 22034529]

15. Morgenstern LB, Staub L, Chan W, et al. Improving delivery of acute stroke therapy: the TLL Temple Foundation Stroke Project. Stroke. 2002; 33:160-66. [PubMed: 11779906]

16. Morgenstern LB, Bartholomew LK, Grotta JC, Staub L, King M, Chan W. Sustained benefit of a community and professional intervention to increase acute stroke therapy. Arch Intern Med. 2003; 163:2198-202. [PubMed: 14557217]

17. Alberts MJ, Latchaw RE, Selman WR, et al. Recommendations for comprehensive stroke centers: a consensus statement from the Brain Attack Coalition. Stroke. 2005; 36:1597-616. [PubMed: 15961715]

18. Scott PA, Frederiksen SM, Kalbfleisch JD, et al. Safety of intravenous thrombolytic use in four emergency departments without acute stroke teams. Acad Emerg Med. 2010; 17:1062-71. [PubMed: 21040107] 
19. Scott PA. Enhancing community delivery of tissue plasminogen activator in stroke through community-academic collaborative clinical knowledge translation. Emerg Med Clin North Am. 2009; 27:115-36. [PubMed: 19218023]

20. Adams H, Adams R, Del Zoppo G, Goldstein LB. Guidelines for the early management of patients with ischemic stroke: 2005 guidelines update a scientific statement from the Stroke Council of the American Heart Association/American Stroke Association. Stroke 2005. 36:916-23.

21. Piriyawat P, Smajsova M, Smith MA, et al. Comparison of active and passive surveillance for cerebrovascular disease: the Brain Attack Surveillance in Corpus Christi (BASIC) Project. Am J Epidemiol. 2002; 156:1062-69. [PubMed: 12446264]

22. Greco PJ, Eisenberg JM. Changing physicians' practices. N Engl J Med. 1993; 329:1271-73. [PubMed: 8413397]

23. Prabhakaran S, McNulty M, O'Neill K, Ouyang B. Intravenous thrombolysis for stroke increases over time at primary stroke centers. Stroke. 2012; 43:875-77. [PubMed: 22135073]

24. Adeoye O, Hornung R, Khatri P, Kleindorfer D. Recombinant tissue-type plasminogen activator use for ischemic stroke in the United States. Stroke. 2011; 42:1952-55. [PubMed: 21636813]

25. Prabhakaran S, McNulty M, O'Neill K, Ouyang B. Intravenous thrombolysis for stroke increases over time at primary stroke centers. Stroke. 2012; 43:875-77. [PubMed: 22135073]

26. Schwamm LH, Fonarow GC, Reeves MJ, et al. Get with the guidelines—stroke is associated with sustained improvement in care for patients hospitalized with acute stroke or transient ischemic attack. Circulation. 2009; 119:107-15. [PubMed: 19075103]

27. The ATLANTIS, ECASS, and NINDS rt-PA Study Group Investigators. Association of outcome with early stroke treatment: pooled analysis of ATLANTIS, ECASS, and NINDS rt-PA stroke trials. Lancet. 2004; 363:768-74. [PubMed: 15016487]

28. The IST-3 collaborative group. The benefits and harms of intravenous thrombolysis with recombinant tissue plasminogen activator within $6 \mathrm{~h}$ of acute ischaemic stroke (the third international stroke trial [IST-3]): a randomised controlled trial. Lancet. 2012; 379:2352-63. [PubMed: 22632908]

29. Dirks M, Niessen LW, van Wijngaarden JD, et al. Promoting thrombolysis in acute ischemic stroke. Stroke. 2011; 42:1325-30. [PubMed: 21393587]

30. Walter S, Kostopoulos P, Haass A, et al. Diagnosis and treatment of patients with stroke in a mobile stroke unit versus in hospital: a randomised controlled trial. Lancet Neurol. 2012; 11:397404. [PubMed: 22497929]

31. Graham GD. Tissue plasminogen activator for acute ischemic stroke in clinical practice: a metaanalysis of safety data. Stroke. 2003; 34:2847-50. [PubMed: 14605319]

32. Wahlgren N, Ahmed N, Davalos A, et al. Thrombolysis with alteplase for acute ischaemic stroke in the Safe Implementation of Thrombolysis in Stroke-Monitoring Study (SITS-MOST): an observational study. Lancet. 2007; 369:275-82. [PubMed: 17258667]

33. Kleindorfer D, Kissela B, Schneider A, et al. Eligibility for recombinant tissue plasminogen activator in acute ischemic stroke: a population-based study. Stroke. 2004; 35:e27-29. [PubMed: 14739423] 


\section{Panel Research in context}

Systematic review

We searched PubMed for trials assessing hospital-based interventions to increase or improve intravenous tissue plasminogen activator (alteplase) use in acute stroke reported before June 1, 2012. We used the search terms "tPA" or "tissue plasminogen activator" or "thrombolysis"; "stroke"; "trial"; and "implementation" or "education". We identified two completed trials: the first was a cluster-randomised, matched-pair, trial (PRomoting ACute Thrombolysis in Ischemic StrokE; PRACTISE) from the Netherlands $;{ }^{29}$ and the second was a quasi-experimental trial from the USA (TLL Temple). ${ }^{15}$ A randomised controlled trial showing the superiority of mobile stroke units in improving time to alteplase decision and treatment was noted but not reviewed, because it focused on prehospital treatment with alteplase with specially equipped mobile stroke units that had CT imaging capabilities-ie, resources not available in the USA. ${ }^{30}$ PRACTISE $^{29}$ compared the effect of an intensive hospital implementation strategy (including forming local teams consisting of a stroke neurologist and a stroke nurse) on increases in alteplase treatment. The primary outcome was treatment with alteplase and the trial was done in 12 hospitals (six pairs), five of which were neurology training sites. Although no significant difference was identified in improvements in overall alteplase use between groups, this result was attributed to low power and effect size because trends favoured the intervention. Analysis of patients admitted within $4 \mathrm{~h}$ of symptom onset showed a significant difference in favour of the intervention. Several observational studies ${ }^{31,32}$ have supported the safety of alteplase use in large cohorts of hospitals but never in a randomly selected population of community hospitals. Other large scale efforts to increase alteplase use have had mixed success. The American Heart Association's Get With The Guidelines experience ${ }^{26}$ supports the value of quality improvement initiatives to improve care after stroke or transient ischaemic attack, such as alteplase delivery. However, generalisation of their findings to the broader hospital population is difficult, because of non-random hospital self-selection and no concurrent control groupstrengths of our study.

Interpretation

Our findings are consistent with those of the PRACTISE trial, ${ }^{29}$ in that the effect size of hospital-based interventions to increase alteplase use seem to be modest overall and might be difficult to detect in cluster-randomised trials of reasonable size. Nevertheless, there does seem to be a signal of efficacy in both trials. Our trial suggests that one approach is effective in community hospitals where neurological resources are frequently scarce. Our findings also clearly establish the safety of thrombolytic delivery in a randomly selected group of emergency departments. Overall, our findings and those of previous studies support further development of educational efforts to increase alteplase use. 


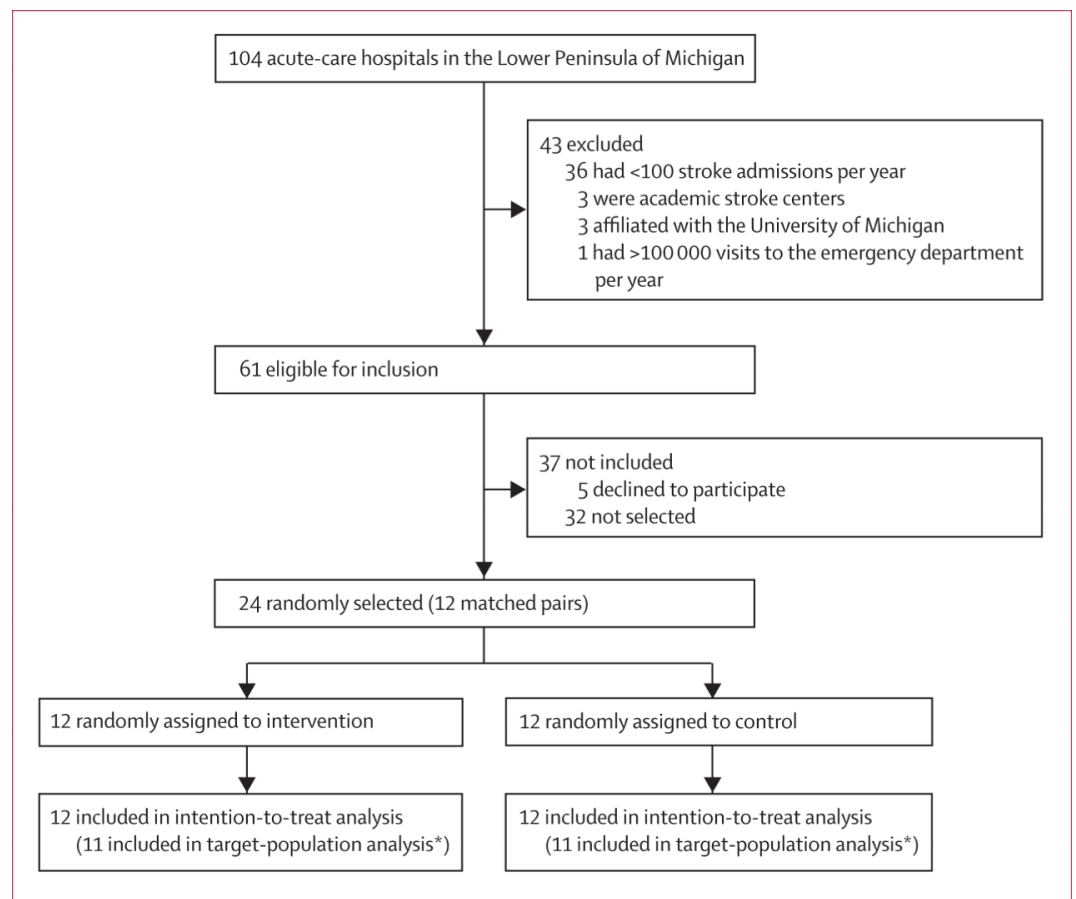

Figure 1.

Trial profile

*One hospital in the control group became an academic comprehensive stroke centre in 2007 after group assignment; this hospital and its matched pair were therefore excluded from the target-population analysis. 


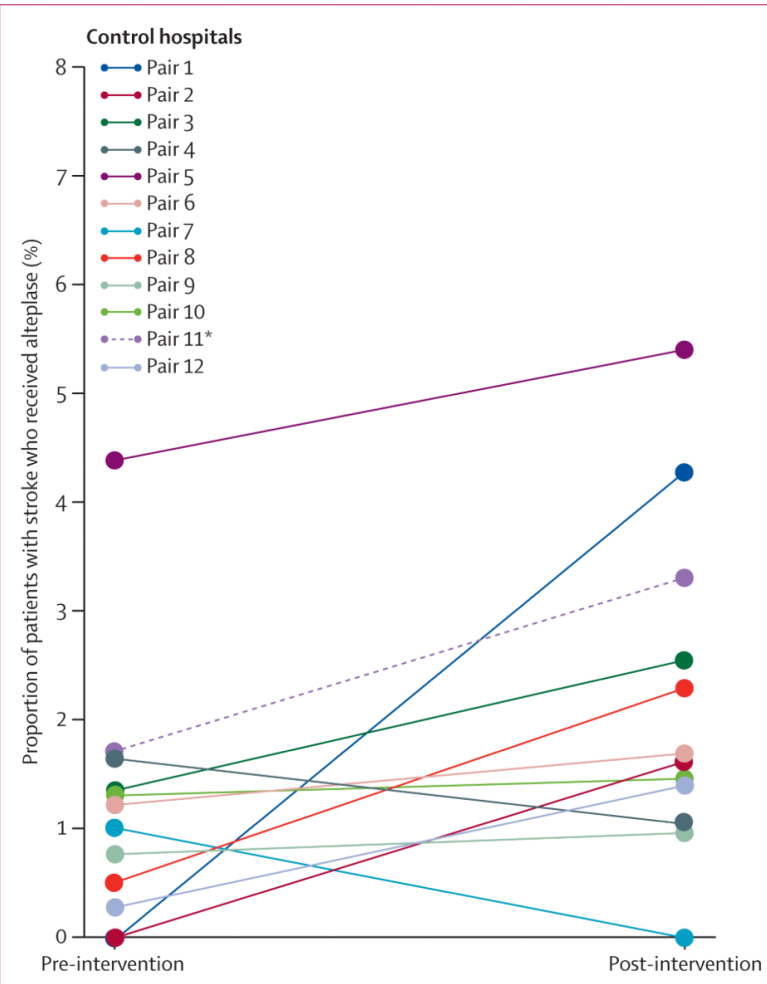

Intervention hospitals

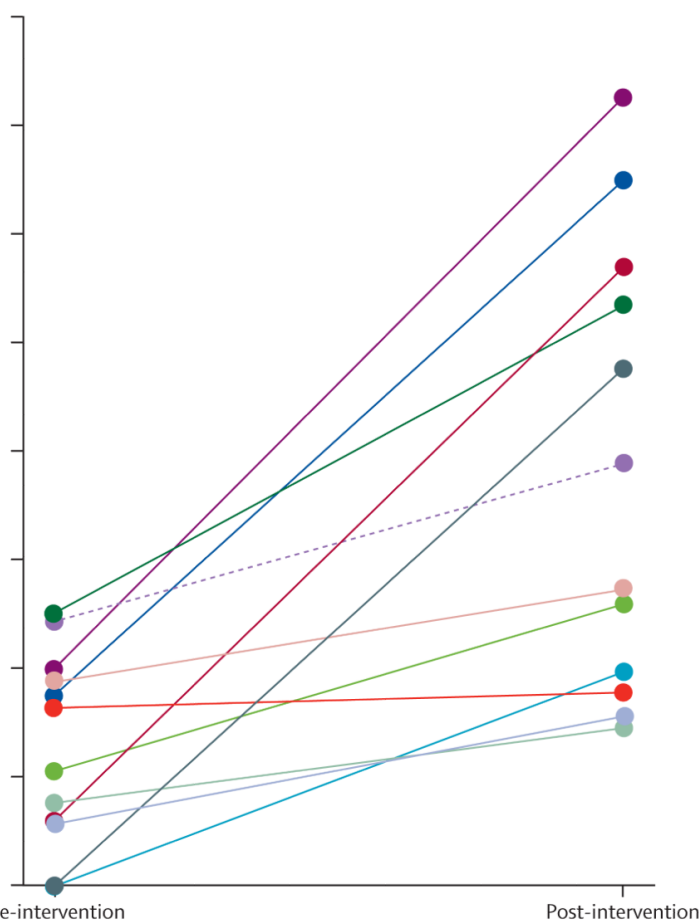

Figure 2.

Change in alteplase use by hospital pair and treatment group *Pair 11 was excluded in the target-population analysis. 
Table 1

Barrier assessment-interactive educational intervention components

Targets

Physicians

nurses;

pharmacists;

administration

teams
Description

1-day meeting of teams from all intervention hospitals (between three and seven people per team) at the coordinating centre.

\section{3 months}

Local barrier assessment

Physicians; nurses;

pharmacists; administration teams
Meeting of the team from (stroke physician and nurse) with individual hospital staff at each intervention hospital. the coordinating centre

\section{6 months}

First on-site educational intervention
Physicians;

nurses;

pharmacists;

EMS;

administration

teams
Mock stroke codes done on-site by coordinating centre personnel. CME lectures delivered on-site by coordinating centre personnel.
Audit and feedback
Physicians;

nurses;

pharmacists; administration teams
Monthly email about success of alteplase treatment at each intervention hospital.

From 6 months to study conclusion

Academic detailing

Physicians

Physicians
Content

$6 \mathrm{~h}$ CME or CEU

educational content about stroke care and behaviour change.

Focus groups to explore local barriers to stroke treatment.

Focus group discussions with emergency physicians and nurses (1 $\mathrm{h}$ each). Structured interviews with neurology, radiology, and hospital administration teams (30 min each). Complete checklist assessment of physical hospital resources for acute stroke treatment.

Mock stroke codes are an actual test of the response and decision making of the stroke chain of survival from EMS pickup, through data collection (CT, labs, etc), to thrombolytic decision; they use the available local resources and are typically not previously announced to the staff. Scenarios are prewritten and use common stroke presentations with variations presented depending on patient management. Patient with stroke is played by local hospital personnel or a mannequin. $2 \mathrm{~h}$ lectures about stroke.

..
Allowed networking between sites to compare barriers and solutions.

Initiated and assisted development of local stroke content experts.

Designed to enhance local identification and understanding of environmental and organisational barriers to stroke treatment.

To further promote local stroke content experts.
After completion, the participants assessed their performance and identified areas for improvement with guidance from the instructor. The lectures aimed to enhance local knowledge about the data for alteplase use in stroke and to address identified specific local barriers.
Allowed anonymous comparison with other intervention sites to promote competition.
Abbreviated form of critical incident stress debriefing. Done for all deviations from treatment guidelines and complications as a result of treatment (eg, intracerebral haemorrhage or serious systemic haemorrhage).
Designed to resolve the emotional content of an event. Provided

professional support in a for local treating

physician(s) with coordinating centre as soon as feasible after identification of index event.

Continuing telephone access to university-based stroke specialist at any time. review of the process leading to the event.

Assisted with decision making about alteplase treatment and follow-up care in real time. 


\begin{tabular}{|c|c|c|c|c|}
\hline & Targets & Description & Content & Purpose \\
\hline Web-based instruments & $\begin{array}{l}\text { Physicians; } \\
\text { nurses; } \\
\text { pharmacists; } \\
\text { administration } \\
\text { teams }\end{array}$ & $\begin{array}{l}\text { Web access to previously } \\
\text { provided CME lectures } \\
\text { and instruments for stroke } \\
\text { treatment (eg, checklists } \\
\text { and protocols). }\end{array}$ & .. & $\begin{array}{l}\text { To serve as a reference } \\
\text { resource and to provide } \\
\text { access to the educational } \\
\text { presentations for those who } \\
\text { could not attend the CME } \\
\text { presentations. }\end{array}$ \\
\hline \multicolumn{5}{|l|}{9 months } \\
\hline $\begin{array}{l}\text { Second on-site educational } \\
\text { intervention }\end{array}$ & $\begin{array}{l}\text { Physicians; } \\
\text { nurses; } \\
\text { pharmacists; } \\
\text { EMS; } \\
\text { administration } \\
\text { teams }\end{array}$ & $\begin{array}{l}\text { Mock stroke codes done } \\
\text { on-site by coordinating } \\
\text { centre personnel. } \\
\text { CME lectures }(2 \mathrm{~h} \text { total }) \\
\text { delivered on-site by } \\
\text { coordinating centre } \\
\text { personnel. }\end{array}$ & As at 6 months. & As at 6 months. \\
\hline \multicolumn{5}{|c|}{ From 12 months to study conclusion } \\
\hline Follow-up interventions & $\begin{array}{l}\text { Physicians; } \\
\text { nurses; } \\
\text { pharmacitsts; } \\
\text { others }\end{array}$ & $\begin{array}{l}\text { Stroke champions meeting } \\
\text { (once a year for } 2 \text { years) } \\
\text { with updated content; } \\
\text { mock stroke code (four } \\
\text { times a year for } 1.5 \text { years). }\end{array}$ & $\begin{array}{l}\text { As at beginning and } 6 \\
\text { months. }\end{array}$ & $\begin{array}{l}\text { To reinforce knowledge } \\
\text { and understanding of the } \\
\text { use of alteplase in stroke. }\end{array}$ \\
\hline
\end{tabular}

$\mathrm{CME}=$ continuing medical education. $\mathrm{CEU}=$ continuing educational unit (the nursing equivalent of $\mathrm{CME}$ ). $\mathrm{EMS}=$ emergency medical services.

* Specific lectures available online. 
Table 2

Hospital characteristics by study phase and group assignment

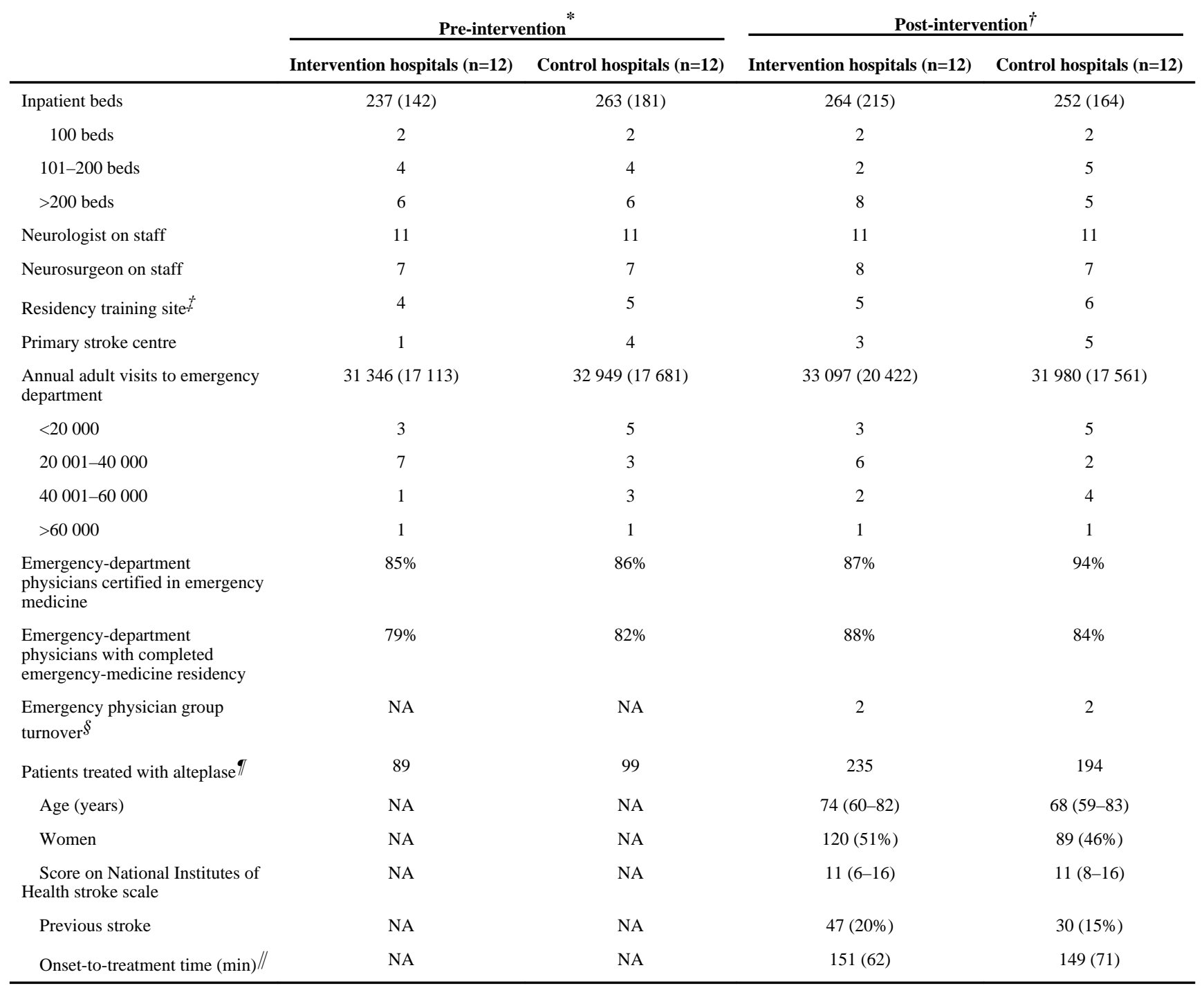

Data are mean (SD), n, \%, median (IQR), or n (\%). Data for intervention period in appendix. NA=not available.

* Pre-intervention data from 2006 .

${ }^{\dagger}$ Post-intervention data from 2008.

${ }^{\dagger}$ Residency training site includes any residency training done on site, irrespective of medical specialty.

$\S_{\text {Indicates loss of hospital contract for group staffing the emergency department. }}$

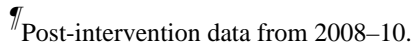

/Data missing for two control patients. 
Table 3

Change in use of alteplase

\begin{tabular}{|c|c|c|c|c|c|}
\hline & $\begin{array}{l}\text { Pre-intervention phase } \\
\text { (24 months) }\end{array}$ & $\begin{array}{l}\text { Intervention phase } \\
\text { (12 months) }\end{array}$ & $\begin{array}{l}\text { Post-intervention phase } \\
\text { (25 months) }\end{array}$ & $\begin{array}{l}\text { Relative risk }(95 \% \\
\text { CI }{ }^{*}\end{array}$ & p value \\
\hline \multicolumn{6}{|c|}{ Intention-to-treat analysis } \\
\hline Control hospitals & $99 / 7946(1.25 \%)$ & $61 / 4212(1.45 \%)$ & $194 / 9222(2.10 \%)$ & .. & \\
\hline Intervention hospitals & $89 / 7119(1.25 \%)$ & $67 / 3905(1.72 \%)$ & $235 / 8419(2.79 \%)$ & $1.37(0.96-1.93)$ & 0.08 \\
\hline \multicolumn{6}{|c|}{ Target-population analysis } \\
\hline Control hospitals & $65 / 5957(1.09 \%)$ & $40 / 3082(1.30 \%)$ & $120 / 6989(1.72 \%)$ & .. & \\
\hline Intervention hospitals & $59 / 5882(1.00 \%)$ & $53 / 3339(1.59 \%)$ & $191 / 7288(2.62 \%)$ & $1.68(1.09-2.57)$ & 0.02 \\
\hline
\end{tabular}

